\title{
Optical Properties of the $(3,12,12)$ Hexagonal Archimedean Photonic Crystal
}

\author{
Đ. Jovanović ${ }^{a, *}$, R. Gajić ${ }^{a}$ And K. HingerL ${ }^{b}$ \\ ${ }^{a}$ Center for Solid State Physics and New Materials, Institute of Physics, Belgrade, Serbia \\ ${ }^{b}$ Zentrum für Oberflächen- und Nanoanalytik, Johannes Kepler Universität, Altenbergerstr. 69, A-4040 Linz, Austria \\ We present a theoretical research of the optical properties of the $(3,12,12)$ hexagonal $2 \mathrm{D}$ Archimedean \\ photonic crystals. The structures are made of GaAs dielectric rods in air. Our research is mainly focused on \\ analyzing symmetry properties of the modes and the appearance of the uncoupled modes which strongly affects \\ the propagation of the wave and the optical performances of the material.
}

PACS numbers: 42.70.Qs, 61.50.Ah, 41.20.Jb, 42.25.Bs, 42.25.Gy

\section{Introduction}

Photonic crystals (PC) are materials whose refractive index is periodically modulated in space which affects emerging photonic band gaps (PBG). In PBG, the existence of any electromagnetic mode is forbidden. On the other hand, there are many phenomena that are not related to band gaps but to specificity of the band structure (for example: negative refraction and light focusing [1-3], self-collimation [4-6]). Furthermore, for solving many phenomena in the $\mathrm{PC}$ it is not enough to know the band structure. We must have some information about symmetry properties of the modes. Precisely, the existence of the band gap is not only the case when, for some frequencies, there is no propagation of the waves. Robertson et al. [7] and later Sakoda [8] theoretically and experimentally proved that beside the symmetric A modes (relative to mirror reflection and the incident plane wave), in the $\mathrm{PC}$, for allowed frequencies, the antisymmetric B modes can also exist for which, unlike A modes, there is no propagation of the wave. They argued that the incident plane wave does not excite these antisymmetric B modes because the coupling of these modes and the incident plane-wave radiation is forbidden by the symmetry (incoming plane wave is even under reflection through the mirror plane and B mode is odd). Ruan et al [9] concluded that in PC for particular frequencies and propagation directions where a $\mathrm{B}$ mode exists the coupling coefficient between a plane wave and the B mode is smaller than $1 \%$ and is about $65 \%$ for the A mode. Therefore, symmetry analysis must be taken into consideration when researching any phenomena related to the propagation of the wave in the PC.

\footnotetext{
* corresponding author; e-mail: djordje@phy.bg.ac.rs
}

In this paper we will classify PC bands according to the group theory by means of the symmetries of the $(3,12,12)$ structure to find symmetric A and especial antisymmetric $\mathrm{B}$ modes which strongly affects the propagation of the wave. Furthermore, we performed calculations of the transmission and reflection to confirm assumptions made according to the symmetry analysis of the modes.

\section{Materials}

We analyzed GaAs 2D photonic crystals dielectric rods $(\varepsilon=12.96)$ in air $(r / a=0.48$, where $r$ is the rod radius and $a$ is the smallest distance between the rods). The structure belongs to class of the Archimedean lattices [10] and by notation of Grünbaum and Shephard [11] is named by $(3,12,12)$. It falls in the hexagonal crystal system with the $p 6 \mathrm{~mm}$ plane symmetry group [12]. Figure 1 presents $(3,12,12)$ lattice, the unit cell and the first Brillouin zone with symmetry points. The first Brillouin zone is the same as the hexagonal (triangular) lattice so we can use the same assumptions for symmetry properties like in well-known hexagonal structure. This is sustained by the fact that symmetry points of our structure belong to the $C_{6 v}, C_{3 v}, C_{2 v}$ point groups for $\Gamma, K$ and $M$ respectively. Moreover, we assumed that our material is homogeneous, linear and lossless.

\section{Analysis}

For obtaining optical properties (response) of the material one must deal with two objectives. One is to determine symmetry properties by calculating band structure and to assign irreducible representations (IREPs) of each band to particular symmetry point and direction (line). The other is to calculate transmission and reflection spectra, by calculating propagation of the wave through the 

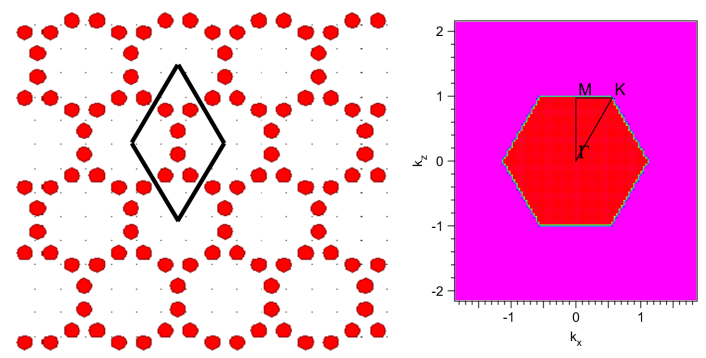

Fig. 1. Crystal lattice, primitive unite cell and the first Brillouin zone with symmetry points of the $(3,12,12)[6]$.

material, in order to confirm the assumptions made in the previous analysis. The theoretical models that are used are well-known plane wave expansion (PWE) and the group theory for the first problem and the finite-difference time-domain (FDTD) for the second.

Briefly, IREPs of the modes can be obtained from investigated symmetry properties of the solutions of the Maxwell equations (scalar fields). More precisely, we must associate symmetries expressed by the symmetry group (point group) with the symmetry properties of the specific field. For detailed explanation of the models for calculating IREPs and symmetry assignment of the bands in hexagonal $(3,12,12)$ and other structures there are many books and papers with all needed tools and tables [13-16].

Figure 2 presents the band structure of the first seven TE polarization bands (TE polarization means that electric field vectors is normal to the plane of the wave propagation) of the $(3,12,12)$ structure with $r / a=0.48$ (the rods almost touch). The model for the calculation is PWE which observe PC as an infinite structure. Furthermore, we assigned symmetry modes obtained by the PWE and the group theory analysis of the symmetry of the TE mode. A and B modes, with or without subscripts, are IREPs of the TE mode in the specific symmetry point or direction respectively. It can be seen that beside the partial band gaps for both symmetry directions $(\Gamma M$ and $\Gamma K)$ there are many antisymmetric B modes (relative to mirror reflection and the incident plane wave) for which there is no coupling with incident wave and therefore no propagation of the wave through the material. Appearance of the B mode is particularly expressed in the $\Gamma K$ symmetry direction because of the existence of the E mode in $\Gamma$ and $\mathrm{K}$ symmetry point (by the group theory double-degenerate E mode splits, for the symmetry direction, in the one non-degenerate A mode and the one non-degenerate B mode). In addition, we know that some specific phenomena that are related for applications in the optics and integrated optics (negative refraction uses for lenses and self-collimation uses for waveguides) emerge in the low frequency bands (in the second mode for TE polarization [6]). Therefore, $\Gamma K$ direction would be useless for those applications.

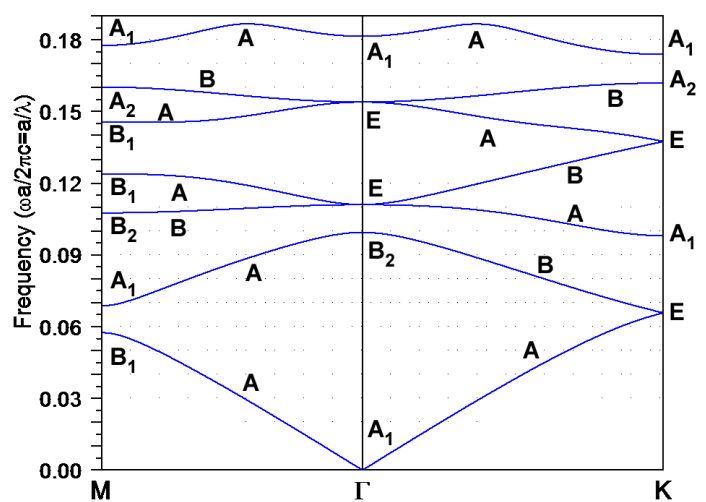

Fig. 2. Band structure with IREPs of the first seven bands, for the TE polarization and $r / a=0.48$.

In order to confirm the predictions obtained by the symmetry analysis we calculate transmission and reflection spectra for $(3,12,12)$ structure, TE polarization, $r / a=0.48$, and $\omega a / 2 \pi c=0.0-0.2$. We use FDTD model for calculation of these spectra. Dimensions of the structure are $6 \times 9$ layers of dodecagons (thickness $\times$ width) which is enough for good calculations. For measuring transmission and backward reflection we put detectors (monitors) near the surface of the PC. In the case of the backward reflection detector is located below the field source. To quantify transmission and reflection we measured the overlap integral

$$
S(t)=\frac{\int_{-\infty}^{+\infty}\left[E_{\mathrm{p}}(t) E_{0}^{*}\left(t_{o}\right)\right] \overline{\mathrm{d} A}}{\int_{-\infty}^{+\infty}\left|E_{0}(t)\right|^{2} \overline{\mathrm{d} A}} .
$$

It consists of the $E_{\mathrm{p}}$ (propagation field at the location of the detector), $E_{0}$ (source field) and the integration is over area $A$ of the detector. The source field is a Gaussian beam wide enough to be considered as a plane wave. The results are presented in Fig. $3 \mathrm{a}$ and $3 \mathrm{~b}$ for $\Gamma M$ and $\Gamma K$ directions, respectively. The obtained spectra were normalized to the source spectra and are labeled according to previous symmetry analysis. We get sharp areas of the high transmittance (low reflectance) for frequencies of the A mode and the opposite where there are PBG or B modes. As explained earlier, B modes mainly emerge for the $\Gamma K$ direction. We get good agreement with results made by symmetry analysis. Exceptions emerge only for A and B mode (third and forth mode) in the $\Gamma M$ direction and the frequency range $0.105-0.120$. The reason is that FDTD model observes a finite structure unlike PWE which assumes an infinite structure so the frequency range for those $\mathrm{A}$ and $\mathrm{B}$ modes obtained with FDTD is narrower from modes obtained by PWE presented in the band structure. In addition, those modes occur between two wide PBG and cannot be well resolved. We can conclude that for $\Gamma M$ symmetry direction our structure has better performance and will be more suitable for applications. 


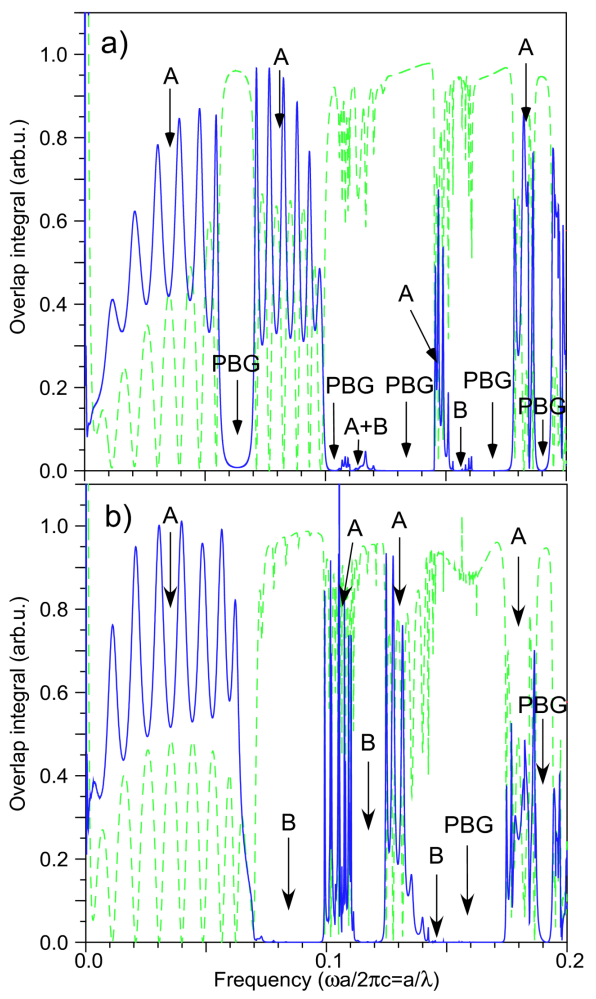

Fig. 3. Transmission (full line) and reflection (dashed line) spectra for the (a) $\Gamma M$ and (b) $\Gamma K$ crystal direction respectively. Spectra are labeled according to the symmetry analysis of the modes.

\section{Conclusions}

We present our research of the optical properties of the $(3,12,12)$ hexagonal 2D Archimedean dielectric PC structure. Optical properties are obtained by analyzing the symmetry properties of the PC modes and the transmission and reflection spectra of the material. We have shown that optical properties of the structure are strongly affected not only with symmetric A modes and PBG but also with antisymmetric B modes that do not couple with incident wave and give no transmission through the structure. This all implies that symmetry analysis must be taken into consideration when researching any phenomena that is connected with the propagation of the wave in the PC. Furthermore, this structure will have better performance and be more suitable for applications for the $\Gamma M$ symmetry direction, since for this direction $\mathrm{B}$ modes have a smaller impact on the optical properties.

\section{Acknowledgments}

This work is supported by the Serbian Ministry of Science under project No. 141047. Đ.J. is grateful to G. Isić to stimulating discussions. R.G. acknowledges support from EU FP7 projects Nanocharm and NIM_NIL. K.H. is grateful to the Austrian NILmeta-NILaustria project from FFG for partial support.

\section{References}

[1] S. Foteinopoulou, C.M. Soukoulis, Phys. Rev. B 67, 235107 (2003).

[2] Đ. Jovanović, R. Gajić, K. Hingerl, Opt. Exp. 16, 4048 (2008).

[3] E. Cubukcu, K. Aydin, E. Ozbay, S. Foteinopoulou, C.M. Soukoulis, Nature 423, 604 (2003).

[4] H. Kosaka, T. Kawashima, A. Tomita, M. Notomi, T. Tamamura, T. Sato, S. Kawakami, Appl. Phys. Lett. 74, 1212 (1999).

[5] P.T. Rakich, M.S. Dahlem, S. Tandon, M. Ibanescu, M. Soljacic, G.S. Petrich, J.D. Joannopoulos, L.A. Kolodziejski, E.P. Ippen, Nature Materials 5, 93 (2006).

[6] Đ. Jovanović, R. Gajić, D. Djokić, K. Hingerl, Acta Phys. Pol. A 116, 55 (2009).

[7] W.M. Robertson, G. Arjavalingam, R.D. Meade, K.D. Brommer, A.M. Rappe, J.D. Joannopoulos, Phys. Rev. Lett. 68, 2023 (1992).

[8] K. Sakoda, Phys. Rev. B 52, 7982 (1995).

[9] Z. Ruan, M. Qiu, S. Xiao, S. He, L. Thylén, Phys. Rev. B 71, 045111 (2005)

[10] J. Kepler, Harmonices Mundi, Linz 1619.

[11] B. Grünbaum, G. Shephard, Tilings and Patterns, Freeman, New York 1987.

[12] T. Hahn, International Tables for Crystallography Volume A: Space-Group Symmetry, Springer, Netherlands 2005, p. 105.

[13] J.F. Cornwell, Group Theory and Electronic Energy Bands in Solids, Selected Topics in Solid State Physics 10, North-Holland, Amsterdam 1969.

[14] J.F. Cornwell, Group Theory in Physics, Techniques in Physics, 7, Academic Press, London 1984.

[15] K. Sakoda, Optical Properties of Photonic Crystals, 2nd ed., Springer-Verlag, Berlin 2005.

[16] W. Hergert, M. Däne, Phys. Status Solidi A 197, 620 (2003). 